\title{
THE DUAL SPACES OF BANACH ALGEBRAS
}

BY

J. M. G. FELL

Introduction. A great deal of work has been done in the last fifteen years on the theory and classification of irreducible unitary representations of locally compact groups. It is also generally recognized (see for example $[12, \S 8])$ that, at least for some classes of groups, it is artificial to confine oneself to unitary representations; one ought to study the irreducible Banach space representations also. One cogent reason for this is that "analytic continuation" of representations inevitably leads us outside the unitary domain. Thus one ought to try to extend to the nonunitary case as much as possible of the theory developed for the unitary situation.

The topology of the dual spaces of $C^{*}$-algebras and locally compact groups (that is, the spaces of their irreducible unitary representations) has been studied in several papers (see $[2],[3],[4],[6])$. In the present paper we begin the study of the topology of the "nonunitary dual spaces" of algebras and groups. Two closely related motives suggest this: First, one would like to generalize the now classical theory of commutative Banach algebras to the noncommutative case even in the absence of an involution - in particular to obtain a noncommutative version of the Banach-algebraic approach to the theory of analytic functions (see [14]). Secondly, one would like to use this to develop an abstract theory of "analytic continuation" of group representations.

Unfortunately, the space of all irreducible Banach space representations of an arbitrary Banach algebra or locally compact group seems too vast and unstructured to submit to a thorough analysis at present. We do not even know what we ought to mean by the equivalence of two such representations. Some "happy restriction" of our subject-matter is called for. The work of Harish-Chandra and Godement [8] suggests what this restriction ought to be. Among Banach algebras $A$, it suggests that we should look first at those whose irreducible representations are all of bounded finite dimension, that is, whose dual spaces are of bounded degree. Among locally compact groups, it suggests that we first examine groups $G$ having a "large" compact subgroup. The group algebra of such a group will have a large stock of subalgebras whose duals are of bounded degree; and the study of the irreducible Banach space representations of $G$ reduces to that of the (finite-dimensional) irreducible representations of these subalgebras. The class of groups having "large" compact subgroups includes all Euclidean

Received by the editors August 21, 1963. 
motion groups and all connected semi-simple Lie groups with finite center.

The present paper examines the topology of the "dual spaces" of complex associative algebras - especially of Banach algebras whose duals are of bounded degree. The application to groups having large compact subgroups will be postponed to a subsequent paper.

What should be the definition of the topological dual space $\hat{A}$ of an arbitrary complex associative algebra $A$ ? The elements of $\hat{A}$ will be representations of $A$ which are in some sense irreducible. In this paper, inspired by Mackey $[12, \S 8]$ we take them to be the "completely irreducible linear system representations" of $A$. This definition has the advantage that an element $T$ of $\hat{A}$ is uniquely determined by the knowledge of one nonzero linear functional on $A$ "associated with" $T$ (see Proposition 2). For the topology of $\hat{A}$ we have at least two choices - the Jacobson "hull-kernel" topology of primitive ideals, or some "functional" topology based on convergence of linear functionals on $A$ associated with the representations. Guided by the Gel'fand topology for the dual space of a commutative Banach algebra, we choose the latter. It will turn out that, for Banach algebras with dual spaces of bounded degree, the functional topology is very natural; it is insensitive to various formal modifications in its definition. The same of course is true for $C^{*}$-algebras (for these, indeed, there is no difference between the functional and hull-kernel topologies, see [4]). For general algebras the functional topology may well be sensitive to small modifications in its definition, and therefore less "natural." However, it will be convenient to have at least a tentative definition available in all cases.

Our paper is divided into two chapters and ten sections. The first chapter is introductory. It consists mostly of fundamental definitions and a few useful general facts about arbitrary untopologized algebras. The second chapter contains our main results on Banach algebras and their representations of bounded dimension. The contents of the sections are as follows:

In $\$ 1$ we define linear system representations and the dual space $\hat{A}$ of $A$. In $\$ 2$ we topologize $\hat{A}$ with the so-called functional topology; and in $\$ 3$ we show that, if $A$ is a $C^{*}$-algebra, the present definition coincides in all respects with the older one. In $\$ 4$ it is shown that, if $B$ is either a twosided ideal of $A$ or a subalgebra of the form $e A e$ ( $e$ being an idempotent), $\hat{B}$ can be identified both setwise and topologically with an open subset of $\hat{A}$. This result will be valuable in the forthcoming application to groups. $\$ 5$ is a brief discussion of the finite-dimensional elements of $\hat{A}$.

Now suppose that $A$ is a Banach algebra, and that $\hat{A}^{(n)}$ is the set of elements of $\hat{A}$ of dimension no greater than the integer $n$. In $\S 7$ we prove essential continuity and boundedness properties of the elements of $\hat{A}^{(n)}$, and the weak * compactness of the set of characters of $A$ of dimension no greater than $n$. \$8 contains two theorems asserting the "naturalness" of 
the topology of $\hat{A}^{(n)}$; one of them connects convergence in $A^{(n)}$ with the convergence of characters. In $\S 9$ we show that $\hat{A}^{(n)}$ shares the general topological properties of the dual spaces of $C^{*}$-algebras. The latter are known to have the Baire property [2], to be locally compact [6], and to satisfy the second axiom of countability if $A$ is separable [5]. Our $\hat{A}^{(n)}$ also has these properties (and of course more special ones also). Finally, let $A$ be a Banach *-algebra and $B$ its $C^{*}$-completion. There is a natural one-to-one correspondence between $\hat{A}_{u n}^{(n)}$ (the set of ${ }^{*}$-representations in $\hat{A}^{(n)}$ ) and $\hat{B}_{u n}^{(n)}$. In $\$ 10$ we show that this correspondence is topological.

The only parts of Chapter I needed in Chapter II are $\$ \$ 1$ and 2 and Remark 1 following Proposition 3 in \$4.

Here are a few points of notation: $\Lambda$ is the void set, $\left\langle x_{1}, \cdots, x_{n}\right\rangle$ denotes sequences, and $(x, y)$ inner products. $\operatorname{Ker}(T)$ is the kernel of $T, \operatorname{Tr}(A)$ the trace of $A$, and $f \mid S$ the restriction of $f$ to $S$.

All linear spaces and algebras are over the complexes. The zero vector in any linear space $H$ is $\theta$; and $H^{\#}$ is the space of all linear functionals on $H$. If $H$ is a Banach space, $H^{\#}$ is the space of continuous linear functionals on $H$. We shall denote by $M_{n}$ the $n \times n$ total matrix algebra over the complexes.

\section{Chapter I}

1. Linear system representations. Throughout this chapter $A$ will be a fixed associative algebra without topology. The reverse algebra of $A$ (with the same set and linear operations but with multiplication reversed) will be called $\widetilde{A}$.

A representation of $A$ in $H$ is a homomorphism $T$ of $A$ into the algebra of all linear endomorphisms of a linear space $H=H(T)$ (the space of $T$ ). By $\operatorname{dim}(T)$ (the dimension of $T$ ) we mean the dimension of $H(T)$. Two representations $T$ and $T^{\prime}$ are equivalent (in symbols, $T \cong T^{\prime}$ ) under $U$ if $U$ is a linear isomorphism of $H(T)$ onto $H\left(T^{\prime}\right)$ such that $T^{\prime}(a)=U T(a) U^{-1}$ for all $a$ in $A$. $T$ is a zero representation if $T(a)=\theta$ for all $a$. If there are no linear subspaces of $H(T)$ stable under all the $T(a)$ other than $\{\theta\}$ and $H(T)$, and if $T$ is not a zero representation, then $T$ is irreducible. Suppose that $H(T) \neq\{\theta\}$, and that, for any positive integer $r$ and any $2 r$ vectors $x_{1}, \cdots, x_{r}, y_{1}, \cdots, y_{r}$ in $H(T)$ such that the $x_{1}, \cdots, x_{r}$ are linearly independent, there is an $a$ in $A$ for which $T(a) x_{i}=y_{i}$ for all $i$; then $T$ is completely irreducible. By the Jacobson Density Theorem [10, p. 28], $T$ is completely irreducible if and only if (a) it is irreducible and (b) the only linear endomorphisms of $H(T)$ which commute with all the $T(a)$ are the complex multiples of the identity operator. An irreducible finite-dimensional representation is completely irreducible by Burnside's Theorem $[1$, Theorem $27.4]$.

Sometimes we shall speak of irreducibility and equivalence of invariant 
subspaces of a representation $T$, referring of course to the restrictions of $T$ to these subspaces.

A duality between two linear spaces $H_{1}$ and $H_{2}$ is a complex bilinear functional $\langle x, y\rangle \rightarrow(x \mid y)$ on $H_{1} \times H_{2}$ such that (a) if $x \in H_{1}$ and $(x \mid y)$ $=0$ for all $y$ in $H_{2}$, then $x=\theta$, and (b) if $y \in H_{2}$ and $(x \mid y)=0$ for all $x$ in $H_{1}$, then $y=\theta$. A linear system is a pair $\left\langle H_{1}, H_{2}\right\rangle$ of linear spaces together with a duality between them (which will usually be written $(x \mid y)$ without explicit mention). Two linear systems $H=\left\langle H_{1}, H_{2}\right\rangle$ and $K$ $=\left\langle K_{1}, K_{2}\right\rangle$ are isomorphic if there are linear isomorphisms $F_{i}$ of $H_{i}$ onto $K_{i}(i=1,2)$ such that $(x \mid y)=\left(F_{1}(x) \mid F_{2}(y)\right)$ for all $x$ in $H_{1}$ and $y$ in $H_{2}$; such a pair $F=\left\langle F_{1}, F_{2}\right\rangle$ is an isomorphism of $H$ and $K$.

If $H$ is a linear space and $K$ is a total subspace of $H^{\#},\langle H, K\rangle$ is a linear system under the natural duality $(x \mid \phi)=\phi(x)$. Conversely, every linear system is of this form (to within isomorphism). A finite-dimensional space $H$ is the first space of an essentially unique linear system, namely $\left\langle H, H^{\#}\right\rangle$.

By a linear system representation of $A$ we mean a linear system $\left\langle H_{1}, H_{2}\right\rangle$, together with a representation $T_{1}$ of $A$ in $H_{1}$ and a representation $T_{2}$ of $\widetilde{A}$ in $H_{2}$, such that $\left(T_{1}(a) x \mid y\right)=\left(x \mid T_{2}(a) y\right) \quad\left(a \in A, x \in H_{1}, y \in H_{2}\right)$. We shall frequently write $T$ for the pair $\left\langle T_{1}, T_{2}\right\rangle$ and $H(T)$ for the linear system $\left\langle H_{1}, H_{2}\right\rangle$. Two linear system representations $S$ and $T$ of $A$ are equivalent (in symbols, $S \cong T$ ) under $F$ if $F$ is an isomorphism of $H(S)$ and $H(T)$ such that $S_{i} \cong T_{i}$ under $F_{i}(i=1,2)$. When no ambiguity can arise, we shall fail to distinguish between a linear system representation and the equivalence class to which it belongs.

A linear system representation $T$ is irreducible (resp. completely irreducible) if both $T_{1}$ and $T_{2}$ are irreducible (resp. completely irreducible). We write $\operatorname{dim}(T)$ for the dimension of $T_{1}$ (or of $T_{2}$ ) if this is finite.

By the kernel $\operatorname{Ker}(T)$ of a linear system representation $T=\left\langle T_{1}, T_{2}\right\rangle$ of $A$ we shall mean $\operatorname{Ker}\left(T_{1}\right)$ (or $\operatorname{Ker}\left(T_{2}\right)$, which is the same).

Definition. Throughout this paper $\hat{A}$ will denote the family of all equivalence classes of completely irreducible linear system representations of $A$. By $\hat{A}^{f}$ (resp. $\hat{A}^{(n)}$, resp. $\hat{A}_{n}$ ) we mean the subset of $\hat{A}$ consisting of those $T$ such that $\operatorname{dim}(T)<\infty$ (resp. $\operatorname{dim}(T) \leqq n, \operatorname{resp} . \operatorname{dim}(T)=n)$.

If $T$ is a representation of $A, T^{\#}$ the adjoint representation of $T$ in $H(T)^{\#}$ (defined by $\left.\left(T^{\#}(a) \phi\right)(x)=\phi(T(a) x)\right), K$ a total linear subspace of $H(T)$ \# stable under $T^{\#}$, and $S(a)=T^{\#}(a) \mid K$, then $\langle T, S\rangle$ is a linear system representation of $A$; and conversely, every linear system representation is equivalent to one of this form. If the representation $T$ is finite-dimensional, $\left\langle T, T^{\#}\right\rangle$ is the linear system representation associated with $T$. We have already seen that in that case $T$ is irreducible if and only if $\left\langle T, T^{\#}\right\rangle$ is completely irreducible. The equivalence of two finite-dimensional repre- 
sentations is the same as equivalence of their associated linear system representations. Thus we may, and shall, identify $\hat{A}^{f}$ with the family of all equivalence classes of finite-dimensional irreducible representations of $A$.

For each $i$ in an index set $I$, let $T^{(i)}$ be a linear system representation of $A$. Let $T_{1}$ and $T_{2}$ be the direct sums of the $T_{1}^{(i)}$ and $T_{2}^{(i)}$ respectively (acting in the direct sum spaces $H\left(T_{1}\right)=\sum_{i} \oplus H\left(T_{1}^{(i)}\right)$ and $H\left(T_{2}\right)=\sum_{i} \oplus H\left(T_{2}^{(i)}\right)$, consisting of functions on $I$ which are $\theta$ in all but finitely many places). If we equip $\left\langle H\left(T_{1}\right), H\left(T_{2}\right)\right\rangle$ with the natural duality $(x \mid y)=\sum_{i}\left(x_{i} \mid y_{i}\right), T$ $=\left\langle T_{1}, T_{2}\right\rangle$ becomes a linear system representation in $\left\langle H\left(T_{1}\right), H\left(T_{2}\right)\right\rangle$, called the direct sum of the $T^{(i)}$, and written $T=\sum_{i} \oplus T^{(i)}$. The direct sum of $\alpha$ copies of a linear system representation $S$ will be written $\alpha \cdot S$.

There is a standard method for constructing a linear system representation $S^{\phi}$ of $A$ from an arbitrary element $\phi$ of $A^{\#}$. Let

$$
\begin{array}{ll}
J_{1}=\{a \in A \mid \phi(b a)=0 & \text { for all } b \text { in } A\}, \\
J_{2}=\{b \in A \mid \phi(b a)=0 & \text { for all } a \text { in } A\} .
\end{array}
$$

Clearly $J_{1}$ and $J_{2}$ are a left ideal and a right ideal of $A$ respectively; and $H_{1}=A / J_{1}$ and $H_{2}=A / J_{2}$ are in duality under the bilinear form

$$
\left(a+J_{1} \mid b+J_{2}\right)=\phi(b a) .
$$

If $S_{1}$ and $S_{2}$ denote the natural representation of $A$ in $H_{1}$ and of $\widetilde{A}$ in $H_{2}$ respectively, then $S^{\phi}=\left\langle S_{1}, S_{2}\right\rangle$ is a linear system representation of $A$ in $\left\langle H_{1}, H_{2}\right\rangle ; S^{\phi}$ will be said to be derived from $\phi$.

If $T=\left\langle T_{1}, T_{2}\right\rangle$ is a linear system representation of $A$, we shall define $\Phi(T)$ to be the linear subspace of $A^{\#}$ spanned by the set of all functionals on $A$ of the form $a \rightarrow\left(T_{1}(a) x \mid y\right)$, where $x \in H\left(T_{1}\right), y \in H\left(T_{2}\right)$. An element $\phi$ of $A^{\#}$ is associated with $T$ if $\phi \in \Phi(T)$. The following lemma is evident:

LEMMA 1. If $\phi$ is associated with $T$, the functionals $a \rightarrow \phi(a b)$ and $a \rightarrow \phi(b a)$ are associated with $T$ for every $b$ in $A$.

Suppose now that $T$ is a completely irreducible linear system representation of $A$. Then the linear map of $H\left(T_{1}\right) \otimes H\left(T_{2}\right)$ onto $\Phi(T)$ which sends $x \otimes y$ into the functional $a \rightarrow\left(T_{1}(a) x \mid y\right)$ is one-to-one. It follows that each $\phi$ in $\Phi(T)$ can be written in the form

$$
\phi(a)=\sum_{i=1}^{r}\left(T_{1}(a) x_{i} \mid y_{i}\right)
$$

where the $x_{1}, \cdots, x_{r}$ are linearly independent in $H\left(T_{1}\right)$, the $y_{1}, \cdots, y_{r}$ are linearly independent in $H\left(T_{2}\right)$, and the $r$ is unique. We call $r$ the rank of $\phi$ (relative to $T$ ).

Proposition 1. If $T \in \hat{A}$ and $\phi$ is an element of $\Phi(T)$ of rank $r$ (relative to $T$ ), then $S^{\phi}$ is equivalent to $r \cdot T$. 
Proof. Let $\phi$ be given by (1), where the $x_{i}$ are linearly independent and likewise the $y_{i}$. Constructing $J_{1}$ and $J_{2}$ as in the definition of $S^{\phi}$ we verify (from the complete irreducibility of $T$ ) that

$$
\begin{array}{ll}
J_{1}=\left\{a \mid T_{1}(a) x_{i}=0\right. & \text { for all } i\}, \\
J_{2}=\left\{a \mid T_{2}(a) y_{i}=0\right. & \text { for all } i\} .
\end{array}
$$

Hence $F_{1}$ and $F_{2}$, defined by

$$
\begin{aligned}
& F_{1}\left(a+J_{1}\right)=\left\langle T_{1}(a) x_{1}, \cdots, T_{1}(a) x_{r}\right\rangle, \\
& F_{2}\left(b+J_{2}\right)=\left\langle T_{2}(b) y_{1}, \cdots, T_{2}(b) y_{r}\right\rangle,
\end{aligned}
$$

are one-to-one maps of $A / J_{1}$ onto $H_{1} \oplus \ldots \oplus H_{1}$ and of $A / J_{2}$ onto $\mathrm{H}_{2} \oplus \ldots \oplus \mathrm{H}_{2}$ respectively. It is now completely straightforward to verify that $S^{\phi} \cong r \cdot T$ under $F$.

Proposition 2. If $S$ and $T$ are inequivalent elements of $\hat{A}, \Phi(S) \cap \Phi(T)$ $=\{\theta\}$.

Proof. Suppose that $\theta \neq \phi \in \Phi(T) \cap \Phi(S)$, and that $\phi$ is expressed in the form (1). Choosing $b$ in $A$ so that $T_{1}(b) x_{1}=x_{1}$ and $T_{1}(b) x_{i}=\theta$ for $i>1$, we see that the functional $\psi(a)=\phi(a b)$ belongs to $\Phi(T)$ and is of rank 1 relative to $T$. Also, by Lemma $1, \psi$ belongs to $\Phi(S)$ and has some rank $k>0$ relative to $S$. So, by Proposition $1, S^{\psi} \cong T$ and also $S^{\psi} \cong k \cdot S$. But $T$ is irreducible; so $k=1$ and $S \cong T$. This contradicts the hypothesis.

Proposition 2 says that an element of $\hat{A}$ is uniquely determined by the knowledge of one nonzero functional associated with it.

2. The functional topology of $\hat{A}$. If $L \subset A^{\#}, L^{-}$will be the closure of $L$ in $A^{\#}$ with respect to the topology of pointwise convergence on $A$.

If $\mathscr{S} \subset \hat{A}$, we write $\Phi(\mathscr{S})$ for $\bigcup_{S \in \mathscr{S}} \Phi(S)$.

Lemma 2. Suppose that $T \in \hat{A}, / \mathscr{S} \subset \hat{A}$, and some nonzero element $\phi$ of $\Phi(T)$ belongs to $\Phi(\mathscr{S})^{-}$. Then $\Phi(T) \subset \Phi(\mathscr{S})^{-}$.

Proof. Let $\psi \in \Phi(T)$. By the complete irreducibility of $T$ there are elements $b_{1}, \cdots, b_{r}, c_{1}, \cdots, c_{r}$ of $A$ such that $\psi(a)=\sum_{i=1}^{r} \phi\left(b_{i} a c_{i}\right)(a \in A)$. If $\phi_{\nu} \rightarrow \phi$ pointwise on $A$, where each $\phi_{\nu} \in \Phi(\mathscr{S})$, then $\psi_{\nu} \rightarrow \psi$, where the functionals $\psi_{\nu}$ are defined by $\psi_{\nu}(a)=\sum_{i=1}^{r} \phi_{\nu}\left(b_{i} a c_{i}\right)$ and belong to $\Phi(\mathscr{S})$ by Lemma 1 . Thus $\psi \in \Phi(\mathscr{Y})^{-}$.

Definition. By the functional topology of $\hat{A}$ we mean that topology in which an element $T$ of $\hat{A}$ belongs to the closure of a subset $\mathscr{S}$ of $A$ if and only if $\Phi(T) \subset \Phi(\mathscr{S})$.

By Lemma 2 this closure operation does indeed define a topology. In fact, $T \in \overline{\mathscr{S}}_{\text {if }}$ and only if some nonzero $\phi$ in $\Phi(T)$ lies in $\Phi(\mathscr{S})^{-}$.

If $\theta \neq \phi \in \Phi(\hat{A})$, let us for the moment denote by $Z^{\phi}$ the unique element of $\hat{A}$ with which $\phi$ is associated (see Proposition 2). Then Lemma 2 save 
that the map $\phi \rightarrow Z^{\phi}$ of $\Phi(\hat{A})-\{\theta\}$ onto $\hat{A}$ is continuous and open (with respect to the topology of pointwise convergence on $A$ relativized to $\Phi(\hat{A})$ $-\{\theta\}$, and the functional topology of $\hat{A}$ ).

Definition. The space $\hat{A}$, equipped with the functional topology, will be called the dual space of the algebra $A$.

In the future $\hat{A}$ will always be assumed to carry the functional topology.

In general the space $\hat{A}$ has no separation properties at all. If $S$ and $T$ are in $\hat{A}$, one verifies easily that $S$ belongs to the closure of $\{T\}$ if and only if $\operatorname{Ker}(T) \subset \operatorname{Ker}(S)$. It follows that $S$ and $T$ are distinguished by the topology if and only if $\operatorname{Ker}(S) \neq \operatorname{Ker}(T)$. Thus the space $\hat{A}$ is a $T_{0}$-space if and only if each element of $\hat{A}$ is uniquely determined by its kernel.

3. The dual space of a $C^{*}$-algebra. In this section we shall assume that $A$ is a $C^{*}$-algebra, and shall show that the above definition of the dual space $\hat{A}$ coincides both setwise and topologically with that studied in [4].

Of course, when speaking of $\hat{A}$ we are thinking of $A$ as merely an algebra, without norm or involution.

If $H$ is any Hilbert space, let $\bar{H}$ be the adjoint Hilbert space. That is, $\bar{H}$ has the same underlying set and addition as $H$; and scalar multiplication and inner product are given by $(\lambda \cdot x)_{\bar{H}}=(\bar{\lambda} \cdot x)_{H},(x, y)_{\bar{H}}=(y, x)_{H}$.

Let $T$ be a topologically irreducible ${ }^{*}$-representation of $A$ in a Hilbert space $H$; and let $T^{*}$ be the adjoint of $T$, that is, the *-representation of the reverse $C^{*}$-algebra $\widetilde{A}$ acting in $\bar{H}$ and given by $T^{*}(a)=T\left(a^{*}\right)$. By Kadison's Irreducibility Theorem [11], $T$ and $T^{*}$ are (algebraically) irreducible - in fact, completely irreducible. Hence, with the inner product duality $\langle x, y\rangle$ $\rightarrow(x, y)$ between $H$ and $\bar{H}$, the pair $S=\left\langle T, T^{*}\right\rangle$ constitutes an element of $\hat{A}$ (in the sense of this paper), which we shall describe as coming from the original *-representation $T$.

Lemma 3. The equivalence class of $\left\langle T, T^{*}\right\rangle$ uniquely determines the unitary equivalence class of $T$.

Proof. By [11], the algebraic equivalence class of $T$ determines its unitary equivalence class.

Lemma 4. Every element $S=\left\langle S_{1}, S_{2}\right\rangle$ of $\hat{A}$ comes from some irreducible *-representation $T$ of $A$ in a Hilbert space.

Proof. By [11], every algebraically irreducible algebra-representation of $A$ is algebraically equivalent to a ${ }^{*}$-representation in a Hilbert space. Hence we may assume that $S_{1}$ and $S_{2}$ are actually irreducible ${ }^{*}$-representations of $A$ and $\widetilde{A}$ respectively. If $T_{1}=S_{1}$ and $T_{2}=S_{2}^{*}$, then $T_{1}$ and $T_{2}$ are irreducible *-representations of $A$ acting in Hilbert spaces $H_{1}$ and $H_{2}$, and the duality belonging to $S$ becomes a nonzero conjugate-bilinear functional $\beta$ on $H_{1} \times H_{2}$ satisfying 


$$
\beta\left(T_{1}(a) x, y\right)=\beta\left(x, T_{2}\left(a^{*}\right) y\right)
$$

$\left(a \in A, x \in H_{1}, y \in H_{2}\right)$.

Suppose now that $T_{1}$ and $T_{2}$ are not unitarily equivalent. Then, for any $x$ in $H_{1}$ and $y$ in $H_{2}$, Corollary 7 of [7] asserts that there is a Hermitian element $a$ of $A$ such that $T_{1}(a) x=x, T_{2}(a) y=2 y$. Substituting this in (2), we find that $\beta(x, y)=2 \beta(x, y)$, or $\beta(x, y)=0$, for all $x$ and $y$. But $\beta$ was not identically zero. So $T_{1}$ and $T_{2}$ are unitarily equivalent. Hence without loss of generality we may assume that $H_{1}=H_{2}=H, T_{1}=T_{2}=T$. Let $x$ and $y$ be orthogonal elements of $H$. Since there is a Hermitian operator carrying $x$ into $x$ and $y$ into $2 y,[11]$ gives us a Hermitian element $a$ of $A$ such that $T(a) x=x, T(a) y=2 y$. Substituting this in (2) as before, we find that $\beta(x, y)$ $=0$. From this and the conjugate-bilinearity of $\beta$ it is easily seen that $\beta$ is a constant multiple of the inner product on $H$.

Therefore the element $S$ of $\hat{A}$ is equivalent to that coming from $T$.

Theorem 1. Let $D$ be the dual space of a $C^{*}$-algebra $A$ in the sense of [4], equipped with the hull-kernel topology as defined there. The mapping which assigns to each $T$ in $D$ the element of $\hat{A}$ which comes from $T$ is a homeomorphism of $D$ onto $\hat{A}$.

Proof. By Lemmas 3 and 4 the mapping is one-to-one and onto $\hat{A}$. That it is a homeomorphism follows from Theorem 1.2 of [4] (see especially (ii) of that theorem).

4. Block subalgebras. In this section $A$ is once more an arbitrary complex associative algebra.

A linear system representation $T=\left\langle T_{1}, T_{2}\right\rangle$ of $A$ is nondegenerate if, for $i=1,2$, the linear span of the ranges of the $T_{i}(a)(a \in A)$ is $H\left(T_{i}\right)$. This implies that, if $x \in H\left(T_{1}\right)$ and $T_{1}(a) x=\theta$ for all $a$, then $x=\theta$; and similarly for $H\left(T_{2}\right)$.

Lemma 5. Let $I$ be a two-sided ideal of $A$, and $T=\left\langle T_{1}, T_{2}\right\rangle$ a nondegenerate linear system representation of $I$. Then there exists a unique linear system representation $T^{\prime}$ of $A$ which acts in the same linear system as $T$ and coincides with $T$ on $I$.

Proof. Suppose $a \in A$. If $x_{1}, \cdots, x_{r} \in H\left(T_{1}\right), b_{1}, \cdots, b_{r} \in I$ and

$$
\sum_{i=1}^{r} T_{1}\left(b_{i}\right) x_{i}=\theta
$$

then for each $c$ in $I$

$$
T_{1}(c)\left[\sum_{i=1}^{r} T_{1}\left(a b_{i}\right) x_{i}\right]=T_{1}(c a)\left[\sum_{i=1}^{r} T_{1}\left(b_{i}\right) x_{i}\right]=\theta .
$$

By the sentence preceding this lemma, this implies that $\sum_{i=1}^{r} T_{1}\left(a b_{i}\right) x_{i}=\theta$. 
Hence we may legitimately define $T_{1}^{\prime}(a)$ on $H\left(T_{1}\right)$ by setting

$$
T_{1}^{\prime}(a)\left[\sum_{i=1}^{r} T_{1}\left(b_{i}\right) x_{i}\right]=\sum_{i=1}^{r} T_{1}\left(a b_{i}\right) x_{i}
$$

whenever $x_{1}, \cdots, x_{r} \in H\left(T_{1}\right), b_{1}, \cdots, b_{r} \in I$. Clearly $T_{1}^{\prime}$ is a representation of $A$ in $H\left(T_{1}\right)$ extending $T_{1}$. Similarly $T_{2}^{\prime}$, defined by

$$
T_{2}^{\prime}(a)\left[\sum_{i=1}^{r} T_{2}\left(b_{i}\right) y_{i}\right]=\sum_{i=1}^{r} T_{2}\left(b_{i} a\right) y_{i}
$$

$\left(y_{1}, \cdots, y_{r} \in H\left(T_{2}\right), b_{1}, \cdots, b_{r} \in I\right)$, is a representation of $\widetilde{A}$ in $H\left(T_{2}\right)$ extending $T_{2}$.

If $a \in A, b, c \in I, x \in H\left(T_{1}\right)$, and $y \in H\left(T_{2}\right)$, we have

$$
\begin{aligned}
\left(T_{1}^{\prime}(a) T_{1}(b) x \mid T_{2}(c) y\right) & =\left(T_{1}(a b) x \mid T_{2}(c) y\right)=\left(x \mid T_{2}(c a b) y\right) \\
& =\left(T_{1}(b) x \mid T_{2}(c a) y\right)=\left(T_{1}(b) x \mid T_{2}^{\prime}(a) T_{2}(c) y\right) .
\end{aligned}
$$

Thus $\left\langle T_{1}^{\prime}, T_{2}^{\prime}\right\rangle$ is the required linear system representation of $A$.

The uniquencess of $T^{\prime}$ follows from the fact that any extension of $T$ must satisfy (3) and (4).

Lemмa 6. If $I$ is a two-sided ideal of $A$, and $T$ is a completely irreducible representation of $A$ such that $I \nsubseteq \operatorname{Ker}(T)$, then $T \mid I$ is completely irreducible.

Proof. That $T \mid I$ is irreducible is immediate and well known. Let $M$ be a linear endomorphism of $H(T)$ commuting with $T \mid I$. If $a \in A, b \in I$, and $x \in H(T), \quad M T(a) T(b) x=M T(a b) x=T(a b) M x=T(a) M T(b) x . \quad$ But the $T(b) x$ exhaust $H(T)$. Thus $M T(a)=T(a) M$ for all $a$ in $A$; so $M$ is a scalar operator. It follows that $T \mid I$ is completely irreducible.

For the following proposition, let $I$ be a two-sided ideal of $A$ and put $\hat{A}_{I}=\{S \in \hat{A} \mid I \nsubseteq \operatorname{Ker}(S)\}$. By Lemmas 5 and 6 , the map $S \rightarrow S \mid I$ (by which we mean $\left.\left\langle S_{1}\left|I, S_{2}\right| I\right\rangle\right)$ is a one-to-one map of $\hat{A}_{I}$ onto $\hat{I}$.

Proposition 3. $\hat{A}_{I}$ is an open subset of $\hat{A}$, and the map $S \rightarrow S \mid I$ is a homeomorphism of $\hat{A}_{I}$ onto $\hat{I}$.

Proof. It is evident from the definition of the functional topology that $\hat{A}_{I}$ is open and the map is continuous. To show that $S \mid I \rightarrow S$ is continuous, we have only to observe that, if $\phi$ is a nonzero element of $I^{\#}$ associated with $S \mid I\left(S \in \hat{A}_{I}\right)$, there is a $b_{0}$ in $I$ such that $\psi$, defined on $A$ by $\psi(a)=\phi\left(a b_{0}\right)$, is nonzero and associated with $S$.

REMARK 1. In particular, suppose that $I$ has no unit and $A$ is the algebra obtained by adjoining a unit 1 to $I$. Then $\hat{A}_{I}=\hat{A}-\{u\}$, where $u$ is the linear system representation associated with the homomorphism $a+\lambda \cdot 1 \rightarrow \lambda$ ( $a \in I, \lambda$ complex). Proposition 3 states that $\hat{I}$ can be identified with $\hat{A}-\{u\}$ both setwise and topologically.

REMARK 2. If $I$ is a two-sided ideal of $A$ as before, $(A / I)^{\wedge}$ can obviously 
be identified, both setwise and topologically, with the closed subset $\hat{A}-\hat{A}_{I}$ of $\hat{A}$.

Suppose now that $e$ is a fixed idempotent element of $A$; and put $B=e A e$. We shall show that $\hat{B}$ can be identified both setwise and topologically with an open subset of $\hat{A}$.

If $T$ is any representation of $A$ in a space $H$, let $T^{0}$ be the representation of $B$ in $T(e) H$ given by $T^{0}(b)=T(b) \mid T(e) H(b \in B)$. If $T$ is irreducible (resp. completely irreducible) and $T(e) \neq \theta$, it is easy to see that $T^{0}$ is irreducible (resp. completely irreducible); in this case the equivalence class of $T^{0}$ determines that of $T$. To prove the latter assertion, take a nonzero vector $x$ in $T(e) H$, and observe that $T$ is equivalent to the natural representation of $A$ in $A / J$, where $J=\{a \in A \mid T(a) x=\theta\}=\left\{a \in A \mid T^{0}(\right.$ ecae $) x$ $=\theta$ for all $c$ in $A\}$.

Suppose now that $T=\left\langle T_{1}, T_{2}\right\rangle$ is a linear system representation of $A$. The duality between $H_{1}=H\left(T_{1}\right)$ and $H_{2}=H\left(T_{2}\right)$ is clearly nondegenerate when restricted to $T_{1}(e) H_{1} \times T_{2}(e) H_{2}$. So, with this duality, $\left\langle T_{1}^{0}, T_{2}^{0}\right\rangle$ is a linear system representation of $B$, which we shall call $T^{0}$.

Lемма 7. If $T$ is completely irreducible and $T_{1}(e) \neq \theta$ (or, equivalently, $\left.T_{2}(e) \neq \theta\right)$, then $T^{0}$ is completely irreducible. In this case the equivalence class of $T^{0}$ determines that of $T$. In fact, the mapping $T^{0} \rightarrow T$ is continuous.

Proof. The first statement follows from the preceding paragraph. If $x$ $\in H\left(T_{1}^{0}\right.$ and $y \in H\left(T_{2}^{0}\right)$, then $\left(T_{1}(a) x \mid y\right)=\left(T_{1}^{0}(e a e) x \mid y\right)$. Thus, for each $\phi$ in $\Phi\left(T^{9}\right)$, the functional $a \rightarrow \phi(e a e)(a \in A)$ belongs to $\Phi(T)$. So by Proposition $2 T^{0}$ determines the class of $T$; and the mapping $T^{0} \rightarrow T$ is continuous in virtue of the definition of the functional topology.

Lemma 8. Every element of $\hat{B}$ is of the form $T^{0}$ for some $T$ in $\hat{A}$.

Proof. Let $S=\left\langle S_{1}, S_{2}\right\rangle \in \hat{B}$, with $H\left(S_{i}\right)=K_{i}$. Choose nonzero vectors $x$ in $K_{1}$ and $y$ in $K_{2}$ respectively; and put $\psi(a)=\left(S_{1}(e a e) x \mid y\right) \quad(a \in A)$. Let $T=\left\langle T_{1}, T_{2}\right\rangle=S^{\downarrow}$ (see $\left.\$ 1\right)$.

Thus $T_{1}$ is the natural representation of $A$ in $H_{1}=A / J_{1}$, where $J_{1}$ is the left ideal $\left\{a \in A \mid\left(S_{1}(e c a e) x \mid y\right)=0\right.$ for all $c$ in $\left.A\right\}=\left\{a \in A \mid S_{1}(e c a e) x=\theta\right.$ for all $c$ in $A\}$. Now by the proof of Lemma 8 of [8] we see that $J_{1}$ is a regular maximal left ideal of $A$ whose intersection with $B$ is $\left\{b \in B \mid S_{1}(b) x=\theta\right\}$. It follows that $T_{1}$ is an irreducible representation of $A$ with the property that $T_{1}^{0}=S_{1}$. I claim that $T_{1}$ is completely irreducible. Indeed, let $M$ be a linear endomorphism of $H_{1}$ commuting with $T_{1}$. Then $T_{1}(e) H_{1}$ is stable under $M$, and the restriction $M^{\prime}$ of $M$ to $T_{1}(e) H_{1}$ commutes with $T_{1}^{0}=S_{1}$. But $S_{1}$ is completely irreducible, and therefore $M^{\prime}$ is a scalar $\lambda$ times the identity. Thus, if $x \in T_{1}(e) H_{1}, a \in A$, and $y=T_{1}(a) x$, we have $M y=M T_{1}(a) x=$ $T_{1}(a) M^{\prime} x=\lambda T_{1}(a) x=\lambda y$. So $M$ is a scalar operator, and we have shown 
that $T_{1}$ is completely irreducible. Similarly $T_{2}$ is completely irreducible. It follows that $T$ is an element of $\hat{A}$.

It is easy to see that $\psi \mid B$ is associated with the element $T^{0}$ of $\hat{B}$. On the other hand $\psi \mid B$ is associated with $S$. So by Proposition $2 S \cong T^{0}$, and the lemma is proved.

Thus we have proved all nontrivial parts of the following proposition:

Proposition 4. $\left\{T \in \hat{A} \mid T_{1}(e) \neq \theta\right\}$ is an open subset of $\hat{A}$; call it $\hat{A}_{e}$. The map $T \rightarrow T^{0}$ is a homeomorphism of $\hat{A}_{e}$ onto $\hat{B}$.

There is a somewhat artificial way of combining Propositions 3 and 4 into one. We define a block subalgebra of $A$ as any subalgebra $B$ of $A$ with the following property: It is possible to embed $A$ as a two-sided ideal in a larger algebra $C$, and to find an idempotent element $e$ of $C$ such that $B$ is contained in, and is a two-sided ideal of, $e C e$. Obviously, two-sided ideals and subalgebras $e A e$ (where $e$ is an idempotent in $A$ ) are block subalgebras of $A$. The following theorem follows immediately from Propositions 3 and 4:

Theorem 2. Let $B$ be a block subalgebra of $A$ and put

$$
\hat{A}_{B}=\{T \in \hat{A} \mid B \nsubseteq \operatorname{Ker}(T)\} .
$$

For each $T$ in $\hat{A}_{B}$, and $i=1,2$, the set $K_{i}=\bigcup_{b \in B}\left(\right.$ range $\left.T_{i}(b)\right)$ is a linear subspace of $H\left(T_{i}\right)$, stable and completely irreducible under $T_{i} \mid B$; furthermore the duality is nondegenerate on $K_{1} \times K_{2}$. Let $T^{0}$ denote the element of $\hat{B}$ defined by $T_{i}^{0}(b)=T_{i}(b) \mid K_{i}(i=1,2)$, with the duality obtained by restricting that of $T$. Then the map $T \rightarrow T^{0}$ is a homeomorphism of the (open) subset $\hat{A}_{B}$ of $\hat{A}$ onto $\hat{B}$.

5. Finite-dimensional representations. From this point on, our results concern only finite-dimensional representations. As we have already seen, $\hat{A}^{f}$ can, and will, be identified with the set of all equivalence classes of finitedimensional representations of $A$. In this section we shall mention, partly without proof, some results on $\hat{A}^{f}$ which are valid for quite general $A$. Some of these seem to be of only limited interest, since for Banach algebras they are superseded by the more powerful results of Chapter II.

Proposition 5. If $T \in \hat{A}^{f},\{T\}$ is closed in $\hat{A}$.

Proof. If $T \in \hat{A}^{f}, \Phi(T)$ is a finite-dimensional and hence closed subspace of $A^{\#}$.

Thus $\hat{A}^{f}$, with the relativized functional topology, is a $T_{1}$-space. It need not of course be a Hausdorff space. The standard example is the algebra $A$ (with pointwise operations) of all continuous functions $g$ on $[0,1]$ to $M_{n}$ for which $\lim _{x \rightarrow 0^{+}} g_{i j}(x)=0$ whenever $i \neq j$.

Proposition 6. For each $n=1,2, \cdots, \hat{A}^{(n)}$ is a closed subset of $\hat{A}$. 
Proof. This is most easily proved by the method of polynomial identities. Let $I$ be the intersection of the kernels of all the elements of $\hat{A}^{(n)}$. If $T$ belongs to the closure of $\hat{A}^{(n)}$, then evidently $\operatorname{Ker}(T) \supset I$; so $T$ can be lifted to an element of $(A / I)^{-}$. But, by the method of polynomial identities one shows easily that $A / I$ cannot have any completely irreducible representations of dimension greater than $n$ (see Lemma 1 of $[8]$ ). It follows that $\operatorname{dim}(T) \leqq n$.

We shall omit the proofs of the following three propositions.

Proposition 7. Let $\left\{T^{(i)}\right\}$ be a net of elements of $\hat{A}^{(n)}$ converging to each of the $p$ inequivalent elements $V^{1}, \ldots, V^{p}$ of $\hat{A}$. Then $\sum_{r=1}^{p}\left(\operatorname{dim}\left(V^{r}\right)\right)^{2} \leqq n^{2}$.

REMARK. If $A$ is a Banach algebra, one can assert the stronger conclusion $\sum_{r=1}^{p} \operatorname{dim}\left(V^{\gamma}\right) \leqq n$ in Proposition 7 (see Proposition 13); and it seems likely that the same holds quite generally.

Proposition 7 shows that, though $\hat{A}^{(n)}$ need not be Hausdorff, at least no net of elements of $\hat{A}^{(n)}$ can converge to more than $n^{2}$ distinct elements. It also implies that $\hat{A}_{n}$ is a Hausdorff subspace of $\hat{A}$.

Proposition 8. The map $T \rightarrow \chi_{T}\left(T \in \hat{A}_{n}\right)$ is a homeomorphism of $\hat{A}_{n}$ into $A^{\#}$ (with the pointwise convergence topology for $A^{\#}$ ).

Here $\chi_{T}$ is the character of $T$; that is, $\chi_{T}(a)=\operatorname{Tr}\left(T_{a}\right)$ for all $a$ in $A$.

Proposition 9. Let $T \in \hat{A}_{n}$ and $\mathscr{S} \subset \hat{A}_{n}$. Then $T$ belongs to the closure of $\mathcal{S}$ if and only if there is a matrix representation $Q$ of $A$ equivalent to $T$, and $a$ net $\left\{Q^{(i)}\right\}$ of matrix representations each equivalent to some element of $\mathscr{S}$, such that $\lim _{i} Q_{j k}^{(i)}(a)=Q_{j k}(a) \quad(a \in A, j, k=1, \cdots, n)$.

(By a matrix representation of $A$ equivalent to $Q$ we mean of course a homomorphism of the form $a \rightarrow\left\{Q_{i j}(a)\right\}$, where $\left\{Q_{i j}(a)\right\}$ is the matrix of $Q(a)$ with respect to some fixed basis of the space of $Q$.)

\section{Chapter II}

\section{Preliminaries on matrix algebras.}

Lemma 9. Let $L$ be an n-dimensional complex normed linear space (the norm being \|\|$)$. Then there is a basis $e_{1}, \cdots, e_{n}$ of $L$ such that (i) $\left\|e_{i}\right\|=1$ for all $i$, and (ii) for all complex $\lambda_{1}, \cdots, \lambda_{n}$,

$$
\left\|\sum_{i=1}^{n} \lambda_{i} e_{i}\right\| \geqq 2^{-n+1} \max _{i=1}^{n}\left|\lambda_{i}\right| .
$$

Proof (by induction in $n$ ). It is obvious if $n=1$. Assume it true for dimension $n-1(n \geqq 2)$, and let $M$ be any $(n-1)$-dimensional subspace of $L$. By hypothesis there is a basis $e_{1}, \cdots, e_{n-1}$ of $M$ such that (a) $\left\|e_{i}\right\|=1$ $(i=1, \cdots, n-1)$, and (b) $\left\|\sum_{i=1}^{n-1} \lambda_{i} e_{i}\right\|=2^{-n+2} \max _{i=1}^{n-1}\left|\lambda_{i}\right|$ (for any complex $\left.\lambda_{1}, \cdots, \lambda_{n-1}\right)$. Choose a complex linear functional $\alpha$ on $L$ of norm 1 
whose null space is $M$; and let $e_{n}$ be any vector satisfying $\left\|e_{n}\right\|=1, \alpha\left(e_{n}\right)$ $=1$. It will suffice to show that the basis $e_{1}, \cdots, e_{n}$ has property (ii).

Let $y=\sum_{i=1}^{n} \lambda_{i} e_{i} \in L,\|y\| \leqq 1$. Then $\left|\lambda_{n}\right|=|\alpha(y)| \leqq 1$. Putting $z$ $=\sum_{i=1}^{n-1} \lambda_{i} e_{i}$, we have $\|z\|=\left\|y-\lambda_{n} e_{n}\right\| \leqq\|y\|+\left|\lambda_{n}\right| \leqq 2$. So by property (b) of the $e_{i}, \max _{i=1}^{n-1}\left|\lambda_{i}\right| \leqq 2^{n-1}$, or (since $\left.\left|\lambda_{n}\right| \leqq 1\right) \quad \max _{i=1}^{n}\left|\lambda_{i}\right| \leqq 2^{n-1}$. Since $y$ was an arbitrary element of the unit ball of $L$, property (ii) follows.

REMARK. If $L$ is a real linear space, Lemma 9 can be strengthened by removing the factor $2^{-n+1}$ (see [13]). If it could be removed in the complex case also, some of our later results could be strengthened.

We shall say that two norms \|\| and \|\|$^{\prime}$ on $M_{n}$ are conjugate if there is a nonsingular $g$ in $M_{n}$ such that $\|a\|^{\prime}=\left\|g^{-1} a g\right\|$ for all $a$ in $M_{n}$.

Lemмa 10. Let \|\| be a norm under which $M_{n}$ is a Banach algebra. Then there exists a norm \|\|$^{\prime}$ conjugate to \|\| satisfying:

$$
\left|a_{i j}\right| \leqq 2^{n-1}\|a\|^{\prime} \quad\left(a \in M_{n}, i, j=1, \cdots, n\right) .
$$

Proof. Let $I$ be the left ideal of $M_{n}$ consisting of those matrices which vanish outside the first column; and let $e^{(1)}, \ldots, e^{(n)}$ be the natural basis of $I\left(e_{i j}^{(k)}=\delta_{k i} \delta_{j 1}\right)$. By Lemma 9 there is a nonsingular $n \times n$ matrix $g$ such that, putting $f^{(i)}=\sum_{j=1}^{n} g_{j i} e^{(j)}$, we have

$$
\begin{aligned}
\left\|f^{(i)}\right\| & =1, \\
\left\|\sum_{i=1}^{n} \lambda_{i} f^{(i)}\right\| & \geqq 2^{-n+1} \max _{i=1}^{n}\left|\lambda_{i}\right|
\end{aligned}
$$

(for all complex $\left.\lambda_{1}, \cdots, \lambda_{n}\right)$. Now, if $a \in M_{n}, a f^{(i)}=\sum_{j=1}^{n}\left(g^{-1} a g\right)_{j i} f^{(j)}$. Hence, by (2) and (3),

$$
\|a\| \geqq \max _{i=1}^{n}\left\|a f^{(i)}\right\|=\max _{i=1}^{n}\left\|\sum_{j=1}^{n}\left(g^{-1} a g\right)_{j i} f^{(j)}\right\| \geqq 2^{-n+1} \max _{i, j=1}^{n}\left|\left(g^{-1} a g\right)_{j i}\right| .
$$

Thus $\left|\left(g^{-1} a g\right)_{j i}\right| \leqq 2^{n-1}|| a \|$ for all $a$ in $M_{n}$ and $i, j=1, \cdots, n$. Replacing $a$ by gag $^{-1}$, we obtain the desired result.

REMARK. The preceding result shows that, if \|\| is any Banach algebra norm of $M_{n}$, a suitably chosen conjugate norm of || || will be "not too small" with respect to a fixed standard norm of $M_{n}$. However, the analogous assertion with "not too small" replaced by "not too large" is false. Indeed, given any positive number $k$, however big, there is a Banach algebra norm \| \| for $M_{n}$ satisfying (i) $\|1\|=1$, and (ii) for any idempotent $e$ of $M_{n}$, other than $\theta$ or $1,\|e\| \geqq k$.

To prove this, take any fixed Banach algebra norm \|\|$_{0}$ for $M_{n}$ with $\|1\|_{0}=1$; and let $E$ be the set of idempotents $e$ in $M_{n}$ which are different from $\theta$ and 1 and satisfy $\|e\|_{0} \leqq k$. Then $E$ is a compact set. For each $e$ in $E$ there is a nonsingular matrix $g_{e}$ for which $\left\|g_{e}^{-1} e g_{e}\right\|_{0}>k$; thus $\left\|g_{e}^{-1} e^{\prime} g_{e}\right\|_{0}$ 
$>k$ for all $e^{\prime}$ in some $E$-neighborhood $U_{e}$ of $e$. A finite number of such neighborhoods $U_{e_{1}}, \cdots, U_{e_{r}}$ cover $E$. For each $a$ in $M_{n}$ put

$$
\|a\|=\max \left[\|a\|_{0},\left\|g_{e_{1}}^{-1} a_{e_{1}}\right\|_{0}, \cdots,\left\|g_{e_{r}}^{-1} a g_{e_{r}}\right\|_{0}\right] .
$$

Then the Banach algebra norm || || satisfies (i) and (ii).

\section{Finite-dimensional representations of a Banach algebra.}

Proposition 10. Let $A$ be any Banach algebra. Then every $T$ in $\hat{A}^{f}$ is continuous. In fact, to each $T$ in $\hat{A}_{n}$ ( $n$ being a positive integer), there is a matrix representation $S$ of $A$ equivalent to $T$ for which

$$
\left|S_{i j}(a)\right| \leqq 2^{n-1}\|a\| \quad(a \in A, \bar{i}, j=1, \cdots, n) .
$$

Proof. Let $T$ be in $\hat{A}_{n}$. Let $\theta \neq u \in H(T)$ and put $I=\{a \in A \mid T(a) u=\theta\}$; $I$ is a regular maximal left ideal of $A$, and hence closed. The natural representation of $A$ in $A / I$ is equivalent to $T$, and is continuous by the closedness of $I$ and the finite-dimensionality of $A / I$. Thus $T$ is continuous.

To prove (4), let $K=\operatorname{Ker}(T)$. Since $T$ is continuous, $K$ is closed. Assuming $T$ to be a matrix representation, we can use the algebraic isomorphism $a+K \rightarrow T(a)$ (of $A / K$ onto $M_{n}$ ) to define a Banach algebra norm \|\|$_{1}$ on $M_{n}$ :

$$
\|T(a)\|_{1}=\|a+K\|=\inf _{b \in K}\|a+b\| .
$$

By Lemma 10 there is a nonsingular element $g$ of $M_{n}$ such that

$$
\left|\left(g^{-1} T(a) g\right)_{i j}\right| \leqq 2^{n-1}|| T(a) \|_{1} \quad(a \in A, i, j=1, \cdots, n) .
$$

Combining the last two relations, and setting $S(a)=g^{-1} T(a) g$, we obtain (4).

REmark. A finite-dimensional representation $T$ of a Banach algebra which is not equivalent to a direct sum of irreducible representations need not be continuous. For examplé, let $A$ have trivial multiplication, take any discontinuous linear functional $\phi$ on $A$, and set

$$
T(a)=\left(\begin{array}{cc}
0 & \phi(a) \\
0 & 0
\end{array}\right) .
$$

Proposition 11. If $A$ is a Banach algebra, and $T$ is any n-dimensional representation of $A$, then $\chi_{T}$ is continuous; in fact $\left\|\chi_{T}\right\| \leqq n$.

Proof. In view of the Jordan-Hölder decomposition it is sufficient to prove this for irreducible $T$. Let \|\|$_{1}$ be the Banach algebra norm on $T(A)$ defined by $\|T(a)\|_{1}=\inf _{b \in \operatorname{Ker}(T)}\|a+b\| . A$ well-known general theorem on Banach algebras tells us that the spectral radius $r$ (the largest of the absolute values of the eigenvalues) of $T(a)$ is no greater than $\|T(a)\|_{1}$. So $\left|\chi_{T}(a)\right| \leqq n r$ $\leqq n\|T(a)\|_{1} \leqq n\|a\|$. 
At this point we must introduce some terminology for characters of a complex associative algebra $A$.

A character of $A$ is a linear functional on $A$ of the form

$$
\psi=\sum_{i=1}^{r} n_{i} \chi_{T}^{(i)}
$$

where the $n_{i}$ are non-negative integers, the $T^{(1)}, \cdots, T^{(r)}$ are pairwise inequivalent elements of $\hat{A}^{f}$, and $\chi_{T}$ denotes the character of $T$ (see §5). It is well known that the decomposition (5) of $\psi$ is unique. If $\psi=\chi_{T}$ for some $T$ in $\hat{A}^{f}, \psi$ is irreducible. The integer $n_{i}$ in (5) is the multiplicity of $\chi_{T}^{(i)}$ in $\psi$. We shall write $\operatorname{dim} \psi=\sum_{i=1}^{r} n_{i} \cdot \operatorname{dim}\left(T^{(i)}\right)$. If $\psi_{1}$ and $\psi_{2}$ are characters, we shall say that $\psi_{1} \prec \psi_{2}$ if $\psi_{2}=\psi_{1}+\psi$ for some character $\psi$. This ordering of characters is transitive and antisymmetric.

Let $\mathscr{X}^{(n)}(A)$ be the family of all characters $\psi$ of $A$ for which $\operatorname{dim} \psi \leqq n$. If $A$ is a Banach algebra, Proposition 11 shows that $\mathscr{X}^{(n)}(A)$ is normbounded. In fact, it is compact in the weak* topology (i.e., the topology of pointwise convergence on $A$ ). We shall prove the following even stronger statement:

Proposition 12. Let $A$ be a Banach algebra, $n$ a positive integer, $p$ a nonnegative integer, and $T$ an element of $\dot{A}^{f}$. Then $\left\{\psi \in \mathscr{X}^{(n)}(A) \mid p \cdot \chi_{T} \prec \psi\right\}$ is weakly* compact.

Proof. First we deal with the case $p=0$. Since each $\psi$ in $\mathscr{X}^{(n)}(A)$ is a sum of $n$ characters each of which is either zero or irreducible, it will be sufficient to take a net $\left\{T^{(i)}\right\}$ of elements of $A_{m}(m \leqq n)$, and show that some subnet of $\left\{\chi_{T^{(i)}}\right\}$ converges to an element of $\mathscr{X}^{(m)}(A)$.

By Proposition 10 we are free to assume that $T^{(i)}$ is a matrix representation satisfying $\left|T_{j k}^{(i)}(a)\right| \leqq 2^{m-1}\|a\|(a \in A, j, k=1, \cdots, m)$. But then the $T_{j k}^{(i)}(a)$ are bounded in $i$ for each $j, k$, and $a$; so we can replace $\left\{T^{(i)}\right\}$ by a subnet such that $\lim _{i} T_{j k}^{(i)}(a)=S_{j k}(a)$ (for each $j, k$, and $a$ ), where $S$ is some $m$-dimensional matrix representation of $A$. But then $\chi_{T}{ }^{(i)} \rightarrow_{i} \chi_{S}$; and the proof for $p=0$ follows immediately from the Jordan-Hölder decomposition of $S$.

Suppose now that $p>0$, and that $\left\{\psi_{i}\right\}$ is a. net of elements of $\mathscr{X}^{(n)}(A)$ of the form $\psi_{i}=p \cdot \chi_{T}+\psi_{i}^{\prime}$, where each $\psi_{i}^{\prime}$ is a character (of dimension no greater than $n-p \operatorname{dim}(T))$. By the preceding paragraph $\left\{\psi_{i}^{\prime}\right\}$ has a subnet converging weakly* to a character $\psi^{\prime}$ for which $\operatorname{dim} \psi^{\prime} \leqq n-p \operatorname{dim}(T)$. So $\left\{\psi_{i}\right\}$ has a subnet converging to the character $\psi=p \cdot \chi_{T}+\psi^{\prime}$ belonging to $\mathscr{X}^{(n)}(A)$.

CoRollary 1. If $A$ is a Banach algebra with unit 1, the set of all irreducible characters in $\mathscr{X}^{(n)}(A)$ is weakly* open relative to $\mathscr{X}^{(n)}(A)$. 
Proof. Let $\left\{\psi_{i}\right\}$ be a net of non-irreducible characters in $\mathscr{X}^{(n)}(A)$ converging weakly* to $\psi$ in $\mathscr{X}^{(n)}(A)$. Writing $\psi_{i}=\psi_{i}^{\prime}+\psi_{i}^{\prime \prime}$, where $\psi_{i}^{\prime}$ and $\psi_{i}^{\prime \prime}$ are nonzero characters, by Proposition 12 we can replace $\left\{\psi_{i}\right\}$ by a subnet and assume that $\psi_{i}^{\prime} \rightarrow \psi^{\prime}, \psi_{i}^{\prime \prime} \rightarrow \psi^{\prime \prime}$ weakly*. Since each $\psi_{i}^{\prime}(1)$ and $\psi_{i}^{\prime \prime}(1)$ is a positive integer, $\psi^{\prime}$ and $\psi^{\prime \prime}$ are nonzero characters; so their sum $\psi$ is not irreducible. Thus the non-irreducible elements of $\mathscr{X}^{(n)}(A)$ form a weakly* closed set.

Corollary 2. If $\left\{\chi_{i}\right\}$ and $\left\{\psi_{i}\right\}$ are nets of elements of $\mathscr{X}^{(n)}(A)$ (based on the same directed set) such that (i) $\chi_{i} \prec \psi_{i}$ for each $i$, and (ii) $\chi_{i} \rightarrow \chi$ and $\psi_{i} \rightarrow \psi$ weakly*, then $x \prec \psi$.

This follows immediately from Proposition 12.

\section{The connection between topology and characters.}

Lemma 11. Fix a positive integer $n$, a non-negative integer $r$, and an index set $I$. For each $i$ in $I$, let $a_{i}$ be an element of $M_{n}$ and $\lambda_{i}$ a linear functional on $M_{n}$; and assume that the zero matrix $\theta$ lies in the closure of $\left\{a_{i} \mid i \in I\right\}$. Then either (A) the family of numbers $\left\{\lambda_{i}\left(a_{i}^{p}\right) \mid i \in I, p=r, r+1, \cdots, r+n-1\right\}$ is unbounded, or (B) 0 lies in the closure of $\left\{\lambda_{i}\left(a_{i}^{n+r}\right) \mid i \in I\right\}$.

Proof. Let the characteristic equation of $a_{i}$ be

$$
t^{n}+\sum_{p=1}^{n} \gamma_{p}(i) t^{n-p}=0
$$

Since each matrix satisfies its own characteristic equation, we may substitute $a_{i}$ for $t$ in this equation, multiply through by $a_{i}^{r}$, and then apply $\lambda_{i}$ to all terms, obtaining

$$
\lambda_{i}\left(a_{i}^{n+\eta}\right)+\sum_{p=1}^{n} \gamma_{p}(i) \lambda_{i}\left(a_{i}^{n+r-p}\right)=0 .
$$

Suppose alternative (A) fails. Then the $\lambda_{i}\left(a_{i}^{n+r-p}\right)$ are bounded in $i$ for each $p=1, \cdots, n$. Since $\theta \in\left\{a_{i}\right\}^{-}$, we can find an $i$ making $\gamma_{1}(i), \cdots, \gamma_{n}(i)$ as small as we wish. But then by (6) $\lambda_{i}\left(a_{i}^{n+\eta}\right)$ is as small as we wish, and so (B) holds.

Lemma 12. The linear span of the set of idempotents in $M_{n}$ is all of $M_{n}$.

Proof. If $e^{(i, j)}$ is the matrix having 1 in the $i, j$ place and 0 elsewhere, then $e^{(i, i)}+c e^{(i, j)}$ is idempotent whenever $i \neq j$ and $c$ is complex.

The next theorem will show that, if $A$ is a Banach algebra and we confine our attention to $\hat{A}^{(n)}$ for some fixed $n$, then the functional topology is a very natural one. In the first place, it has an easy description in terms of characters; in the second place, it is insensitive to various changes in its formal definition. 
We fix a Banach algebra $A$ and a positive integer $n$.

Theorem 3. If $T \in \hat{A}^{(n)}$ and $\mathscr{S} \subset \hat{A}^{(n)}$, then the following are equivalent:

(a) $T$ is in the functional closure of $\mathscr{Y}$;

(b) $\chi_{T} \prec \psi$ for some $\psi$ in the weak ${ }^{*}$ closure of $\left\{\chi_{S} \mid S \in \mathscr{S}\right\}$.

Theorem 4. Fix any dense subalgebra $A_{0}$ of $A$; and let $T \in \hat{A}^{(n)}, \mathscr{S} \subset \hat{A}^{(n)}$. Then the following five conditions are equivalent:

(i) $T$ is in the functional closure of $\mathscr{S}$;

(ii) each $\phi$ in $\Phi(T)$ (see $\S 1$ ) is in the weak* closure of $\{\psi \in \Phi(\mathscr{S}) \mid\|\psi\| \leqq k\}$ for some positive $k$ (depending on $\phi$ );

(iii) some nonzero $\phi$ in $\Phi(T)$ can be approached pointwise on $A_{0}$ by elements of $\Phi(\mathscr{S})$;

(iv) to each matrix representation $S$ of $A$ equivlaent to $T$, there is a positive number $k$ and a net $\left\{S^{(i)}\right\}$ of matrix representations of $A$ each of which is equivalent to some element of $\mathscr{S}$ of dimension no less than $\operatorname{dim} T$, such that

$$
\lim _{i} S_{j k}^{(i)}(a)=S_{j k}(a) \quad(a \in A, \quad j, k=1, \cdots, \operatorname{dim} T)
$$

and

$$
\left|S_{j k}^{(i)}(a)\right| \leqq k\|a\| \quad\left(\text { for all } i, a \text { in } A \text { and } j, k=1, \cdots, \operatorname{dim}\left(S^{(i)}\right)\right) ;
$$

(v) there is a matrix representation $S$ of $A$ equivalent to $T$, and a net $\left\{S^{(i)}\right\}$ of matrix representations each equivalent to some $S$ in $\mathscr{S}$, such that $\operatorname{dim}\left(S^{(i)}\right)$ $\geqq \operatorname{dim}(T)$ for all $i$ and $\lim _{i} S_{j k}^{(i)}(a)=S_{j k}(a)$ for all $j, k=1, \cdots, \operatorname{dim} T$, and all $a$ in $A_{0}$.

Proof. Both theorems will be proved together.

Part I. We shall show that (b) (of Theorem 3) implies (iv) (of Theorem 4).

Choose a net $\left\{T^{(i)}\right\}$ of elements of $\mathscr{S}$ of constant dimension $m \leqq n$, and a character $\psi$ such that $\chi_{T} \prec \psi$ and $\chi_{T}^{(i)} \rightarrow \psi$ weakly*. By Proposition 10 we are free to assume that each $T^{(i)}$ is a matrix representation satisfying

$$
\left|T_{j k}^{(i)}(a)\right| \leqq 2^{n-1}\|a\| \quad \text { for each } a, j, k .
$$

Hence we may pass to a subnet and assume

$$
\lim _{i} T_{j k}^{(i)}(a)=Q_{j k}(a) \quad(a \in A, \quad j, k=1, \cdots, m),
$$

where $Q$ is an $m$-dimensional matrix representation of $A$ whose character is $\psi$. Let $U$ be a nonsingular matrix such that the representation $Q^{\prime}$ defined by $Q^{\prime}(a)=U^{-1} Q(a) U^{-}$is of triangular block form as in the Jordan-Hölder decomposition, with representations $S^{(1)}, \ldots, S^{(r)}$ along the diagonal which are either zero or irreducible. Then $\psi=\sum_{p=1}^{r} \chi_{S}^{(p)}$. Since $\chi_{T} \prec \psi, T \cong S^{(p)}$ for some $p$. Permuting rows and columns, we may suppose

$$
T \cong S^{(1)} \text {. }
$$


Now let $T^{\prime(i)}(a)=U^{-1} T^{(i)}(a) U$. By (8) $\lim _{i} T_{j k}^{(i)}(a)=Q_{j k}^{\prime}(a)$ for all $a, j, k$. By (7), the $\left|T_{j k}^{\prime(i)}(a)\right|$ are majorized by $k\|a\|$ for some constant $k$. Hence, in view of (9), (iv) has been established for some matrix representation $S^{(1)}$ equivalent to $T$.

Any other matrix representation $S$ equivalent to $T$ will be of the form $a \rightarrow V^{-1} S^{(1)}(a) V$ for some nonsingular $V$. Let $W$ be the $m \times m$ matrix coinciding with $V$ in its first $\operatorname{dim}(T)$ rows and columns and with the unit matrix elsewhere. Then the matrix representation $a \rightarrow W^{-1} T^{\prime(i)}(a) W$ will satisfy (iv) for $S$. Thus condition (iv) is established.

Part II. We shall show that (iv) $\Rightarrow$ (ii) and (v) $\Rightarrow$ (iii).

Let $S,\left\{S^{(i)}\right\}, k$ be as in (iv). An element $\phi$ of $\Phi(T)$ is of the form $\phi(a)$ $=\sum_{j, k=1}^{m} \lambda_{j k} S_{j k}(a)$ (where the $\lambda_{j k}$ are complex constants and $m=\operatorname{dim} T$ ). Setting $\phi_{i}(a)=\sum_{j, k=1}^{m} \lambda_{j k} S_{j k}^{(i)}(a)$, we have $\phi_{i} \in \Phi(\mathscr{S}), \phi_{i} \rightarrow \phi$ weakly $^{*}$, and $\left\|\phi_{i}\right\| \leqq k \sum_{j, k}\left|\lambda_{j k}\right|$. Thus (ii) holds. The same argument shows that (v) $\Rightarrow$ (iii).

Part III. We shall show that (iii) $\Rightarrow$ (b). (of Theorem 3).

Assume that (iii) holds but that (b) fails. Then there is a nonzero $\phi$ in $\Phi(T)$, a net $\left\{T^{(i)}\right\}$ of elements of $\mathscr{S}$, and for each $i$ an element $\phi_{i}$ of $\Phi\left(T^{(i)}\right)$, such that

$$
\phi_{i}(a) \rightarrow \phi(a) \quad \text { for all } a \text { in } A_{0} .
$$

As in Part I of the proof, we may suppose that each $T^{(i)}$ is an $m$-dimensional matrix representation of $A$ satisfying

$$
\lim _{i} T_{j k}^{(i)}(a)=Q_{j k}(a) \quad(a \in A, \quad j, k=1, \cdots, m),
$$

where $Q$ is an $m$-dimensional triangular block matrix representation with zero or irreducible representations $S^{(1)}, \cdots, S^{(r)}$ along the diagonal.

I claim there is an element $a$ of $A_{0}$ such that

$$
Q(a)=\theta, T(a)=\text { unit matrix. }
$$

Indeed: Since, by assumption, (b) fails, $T$ cannot be equivalent to any $S^{(p)}$. Hence (using the continuity of $T$ and the $S^{(p)}$, and the denseness of $A_{0}$ ) $T \mid A_{0}$ is an irreducible representation of $A_{0}$ not equivalent to any of the irreducible representations $S^{(p)} \mid A_{0}$. Therefore, by the Extended Burnside Theorem [1, Theorem 27.8], there is a $b$ in $A_{0}$ such that $T(b)$ is the unit matrix and $S^{(p)}(b)=\theta$ for all $p$. In particular, $Q(b)$ is triangular with zeros on the diagonal; and so $a=b^{m}$ satisfies (12).

By the above claim and Lemma 12 there is an element $e$ in $A_{0}$ such that

$$
T(e) \text { is idempotent, } \phi(e) \neq 0, Q(e)=\theta \text {. }
$$

If $\lambda_{i}$ is the linear functional on $M_{m}$ such that $\phi_{i}(a)=\lambda_{i}\left(T^{(i)}(a)\right)(a \in A)$, (10) and (13) give us, for each $k=1,2, \cdots$, 


$$
\lambda_{i}\left(\left(T^{(i)}(e)\right)^{k}\right)=\phi_{i}\left(e^{k}\right) \rightarrow \phi\left(e^{k}\right)=\phi(e) \neq 0 .
$$

On the other hand, by (11) and (13), $T^{(i)}(e) \rightarrow_{i} \Theta$. This together with (14) contradicts Lemma 11; and the proof of Part III is complete.

Part IV. The implications (iv) $\Rightarrow(v)$ and (ii) $\Rightarrow$ (iii) are trivial. Thus we have shown that (b) $\Rightarrow$ (iv) $\Rightarrow(\mathrm{v}) \Rightarrow$ (iii) $\Rightarrow$ (b) and also (iv) $\Rightarrow$ (ii) $\Rightarrow$ (iii). Thus (b), (ii), (iii), (iv), and (v) are all equivalent. In particular, the truth or falsity of (iii) and (v) does not depend on the particular $A_{0}$. But if we put $A_{0}=A$, (iii) becomes (i). So (i) is equivalent to all the rest. This completes the proof of both theorems.

Corollary 1. If a Banach algebra $A$ has a unit 1 , each $\hat{A}^{(n)}$ is compact.

Proof. If $\left\{T^{(i)}\right\}$ is a net of elements of $\hat{A}^{(n)}$, Proposition 12 permits us to pass to a subnet such that $\chi_{T^{(i)}} \rightarrow \psi \in \mathscr{X}^{(n)}(A)$. Since each $\chi_{T^{(i)}}(1)$ is a positive integer, $\psi \neq \theta$. Hence $\chi_{S} \prec^{\prime} \psi$ for some $S$ in $\tilde{A}^{(n)}$; and, by Theorem 3 , the implies $T^{(i)} \rightarrow_{i} S$.

Corollary 2. Let $A$ be a Banach algebra and $n$ a positive integer. Then $\hat{A}_{n}$, with the relativized functional topology, is a locally compact Hausdorff space; and $T \rightarrow \chi_{T}$ is a homeomorphism of $\hat{A}_{n}$ into $A^{*}$ (with the weak ${ }^{*}$ topology).

Proof. The second statement follows from Theorem 3 (with the help of Proposition 12). To prove the first statement, we assume without loss of generality (see Remark 1 following Proposition 3 ) that $A$ has a unit element, and then use Proposition 12 and its Corollary 1.

This corollary will be considerably strengthened by Theorem 6 .

REMaRK 1. The argument of Part I of the preceding proof allows us to strengthen Proposition 7 for the case of a Banach algebra as follows:

Proposition 13. If $A$ is a Banach algebra, $n$ is a positive integer, and $\left\{T^{(i)}\right\}$ is a net of elements of $\hat{A}^{(n)}$ converging to the distinct elements $V^{(1)}$, $\ldots, V^{(r)}$ of $\hat{A}$, then $\sum_{p=1}^{r} \operatorname{dim}\left(V^{(p)}\right) \leqq n$.

Proof. As in Part I of the preceding proof we can pass to a subnet and assume that the $T^{(i)}$ are matrix representations all of the same dimension $m \leqq n$, such that $\lim _{i} T_{j k}^{(i)}(a)=S_{j k}(a) \quad(a \in A, j, k=1, \cdots, m)$, where $S$ is some $m$-dimensional matrix representation of $A$. By Theorem 3 each $V^{i}$ has multiplicity at least one in $S$. This gives the required conclusion.

Remark 2. Let $A, A_{0}$, and $n$ be as in Theorem 4. As we have observed from the continuity of the elements of $\hat{A}^{f}$, the map $T \rightarrow T \mid A_{0}$ is a one-to-one map of $\hat{A}^{f}$ onto a subset of $\left(A_{0}\right)^{\wedge}$. The equivalence of (i) and (iii) in Theorem 4 states that, when restricted to $\hat{A}^{(n)}$, this map is a homeomorphism. In fact the reasoning of Part III of the proof of Theorem 4 yields the following further information: 
Proposition 14. The set $W$ of all $T \mid A_{0}$, where $T$ runs over $\hat{A}^{(n)}$, is closed in $\left(A_{0}\right)^{-}$.

Proof. If $S$ belongs to the closure of $W$, there is a nonzero functional $\phi$ on $A_{0}$ associated with $S$, and a net $\left\{\phi_{i}\right\}$ of functionals on $A$ associated with $\hat{A}^{(n)}$, such that $\phi_{i} \rightarrow_{i} \phi$ pointwise on $A_{0}$. The reasoning of Part III now goes through as before.

9. Properties of $\hat{A}^{(n)}$. Let $A$ be a fixed Banach algebra and $n$ a fixed positive integer. This section deals with the topological properties of $\hat{A}^{(n)}$. The complications arise, of course, from the fact that $\hat{A}^{(n)}$ is not in general Hausdorff.

To begin with, we have a finite increasing sequence

$$
\hat{A}^{(0)}=\Lambda \subset \hat{A}^{(1)} \subset \hat{A}^{(2)} \subset \cdots \subset \hat{A}^{(n)}
$$

of closed subsets of $\hat{A}^{(n)}$ such that (by Corollary 2 of Theorems 3 and 4) each $\hat{A}^{(p)}-\hat{A}^{(\dot{p}-1)}$ is a locally compact Hausdorff space. It follows that $\hat{A}^{(n)}$ has the Baire property, that is, no nonvoid open subset $W$ of $\hat{A}^{(n)}$ can be covered by countably many closed sets each of which has void interior. Indeed, if $W$ is such a set, let $p$ be the smallest integer for which $W \subset \hat{A}^{(p)}$. Then $W-\hat{A}^{(p-1)}$ is a nonvoid open subset of the locally compact Hausdorff space $\hat{A}^{(p)}-\hat{A}^{(p-1)}$, and the result follows from the ordinary Baire category theorem.

We are going to show that $\hat{A}^{(n)}$ is locally compact, and satisfies the second axiom of countability if $A$ is separable. For this we must set up a basis for the open subsets of $\hat{A}^{(n)}$.

As before $\mathscr{X}^{(n)}(A)$ will be the set of all characters of $A$ of dimension no greater than $n$. If $T \in \hat{A}^{(n)}$, put

$$
\mathscr{Y}_{T}=\left\{\psi \in \mathscr{X}^{(n)}(A) \mid \chi_{T} \prec \psi\right\} .
$$

By Proposition $12 \mathscr{Y}_{T}$ is weakly* compact. If $W$ is any subset of $\mathscr{X}^{(n)}(A)$ which is open (relative to $\left.\mathscr{X}^{(n)}(A)\right)$, put

$$
\begin{gathered}
U(W)=\left\{S \in \hat{A}^{(n)} \mid \chi_{S} \in W\right\}, \\
U^{\prime}(W)=\left\{S \in \hat{A}^{(n)} \mid \mathscr{Y}_{S} \subset W\right\} .
\end{gathered}
$$

Lemma 13. If $T \in \hat{A}^{(n)}$, the $U(W)$ (where $W$ runs over all $\mathscr{X}^{(n)}(A)$-open sets containing $\mathscr{Y}_{T}$ ) form a basis of neighborhoods of $T$ in $\hat{A}^{(n)}$. The same holds for the $U^{\prime}(W)$.

Proof. Since $U^{\prime}(W) \subset U(W)$, we need only show that (I) each $U^{\prime}(W)$ is a neighborhood of $T$ in $\hat{A}^{(n)}$, and (II) each neighborhood of $T$ contains some $U(W)$. The set $W$ is always assumed to be open in $\mathscr{X}^{(n)}(A)$ and to contain $\mathscr{Y}_{T}$.

(I) If $T$ belonged to the closure of $\hat{A}^{(n)}-U^{\prime}(W)$, we could find a net $\left\{T^{(i)}\right\}$ of elements of $\hat{A}^{(n)}$, and for each $i$ a character $\phi_{i}$, such that 


$$
\begin{aligned}
& \chi_{T^{(i)}} \prec \phi_{i} \notin W \quad \text { for each } i, \\
& \chi_{T^{(i)} \rightarrow \psi} \text { weakly }^{*},
\end{aligned}
$$

where $\psi$ is a character for which $\chi_{T} \prec \psi$. Passing to a subnet we may assume (by Proposition 12) that $\phi_{i} \rightarrow \phi \in \mathscr{X}^{(n)}(A)$. This, (15), (16), and Corollary 2 of Proposition 12 give $\psi \prec \phi$; and hence (since $\left.\chi_{T} \prec \psi\right) \phi \in \mathscr{Y}_{T}$. But this is impossible since $\phi_{i} \rightarrow \dot{\phi}$ and $\phi_{i} \notin W$.

(II) If (II) fails, we may pick from each $U(W)$ an element $T_{W}$ in such a way that the closure of $\left\{T_{W}\right\}$ does not contain $T$. Directing $\{W\}$ by reverse inclusion so that $\left\{T_{W}\right\}$ becomes a net, we can pass to a subnet by Proposition 12 and assume that

$$
\chi_{T_{W}} \rightarrow \psi,
$$

where $\psi \in \mathscr{X}^{(n)}(A)$. Since $\chi_{T_{W}} \in W$ and $\mathscr{Y}_{T}$ is closed, $\psi \in \mathscr{Y}_{T}$, so that (17) implies $T_{W} \rightarrow T$, contradicting the choice of the $T_{W}$.

Theorem 5. If $A$ is a Banach algebra having a dense subset of (infinite) cardinality $\alpha$, and $n$ is a positive integer, then $\hat{A}^{(n)}$ has a basis of open sets of cardinality no greater than $\alpha$. In particular, if $A$ is separable $\hat{A}^{(n)}$ satisfies the second axiom of countability.

Proof. The ball of radius $n$ in $A^{*}$, with the weak* topology, has a basis $\mathscr{B}$ of open sets of cardinality no greater than $\alpha$. Let $\mathscr{F}$ be the family of all finite unions of sets of the form $V \cap \mathscr{X}^{(n)}(A)$, where $V \in \mathscr{B}$. Then the family of all $U(W)$, where $W$ runs over $\mathscr{F}$, has cardinality no greater than $\alpha$, and forms a basis for the open subsets of $\hat{A}^{(n)}$ by virtue of Lemma 13 (and the compactness of the $\mathscr{Y}_{T}$ ).

Lemma 14. Let $T$ be in $A^{(n)}, \psi$ an element of $\mathscr{Y}_{T}$, and $\left\{\psi_{i}\right\}$ a net of elements of $\mathscr{X}^{(n)}(A)$ for which $\psi_{i} \rightarrow \psi$ weakly*. Then there exists a $\phi$ in $\mathscr{Y}_{T}$, a subnet $\left\{\psi_{j}^{\prime}\right\}$ of $\left\{\psi_{i}\right\}$, and for each $j$ an irreducible character $\chi_{j}$ satisfying $\chi_{j} \prec \psi_{j}^{\prime}$, such that $\chi_{j} \rightarrow \phi$ weakly*.

Proof. Each $\psi_{i}$ is of the form $\sum_{p=1}^{n} \psi_{i}^{p}$, where $\psi_{i}^{p}$ is either 0 or an irreducible character. By Proposition 12 we can pass to a subnet and assume

$$
\psi_{i}^{p} \underset{\imath}{\longrightarrow} \psi^{p} \text { weakly* }
$$

where each $\psi^{p}$ is a character and $\sum_{p=1}^{n} \psi^{p}=\psi$. Thus, since $\chi_{T} \prec \psi$, there is a $p$ for which $\chi_{T} \prec \psi^{p}$. In particular $\psi^{p} \neq 0$; so $\psi_{i}^{p}$ is $i$-eventually irreducible. Since $\psi_{i}^{p} \prec \psi_{i}$ and (18) holds, the requirements of the Lemma are satisfied by taking $\chi_{i}=\psi_{i}^{p}, \phi=\psi^{p}$.

Definition (SEe $\mid 6]$ ). A (not necessarily Hausdorff) topological space $X$ is locally compact if every point of $X$ has a basis of compact neighborhoods.

REMARK. A compact non-Hausdorff space is not in general locally com- 
pact. Thus the theorem that follows cannot be proved by appealing to Corollary 1 of Theorems 3 and 4 .

Theorem 6. If $A$ is a Banach algebra and $n$ is a positive integer, $\hat{A}^{(n)}$ is locally compact.

Proof. By Remark 1 following Proposition 3, we may as well assume that $A$ has a unit 1.

Fix an element $T$ of $\hat{A}^{(n)}$ of dimension $p$, and an open neighborhood $W$ of $\mathscr{Y}_{T}$ in $\mathscr{X}^{(n)}(A)$. By Lemma 13 it will be enough to obtain a compact neighborhood of $T$ contained in $U(W)$.

We begin by fixing a compact $\mathscr{X}^{(n)}(A)$-neighborhood $W_{0}$ of $\mathscr{Y}_{T}$ contained in $W$. Let $\mathscr{E}$ be the set of all irreducible characters. If $Z \subset \mathscr{X}^{(n)}(A)$, let $\mathscr{Y}(Z)=\left\{x \in \mathscr{X}^{(n)}(A) \mid \psi \prec \chi\right.$ for some $\psi$ in $\left.Z\right\}$.

We shall define inductively a finite sequence of sets

$$
Q_{p}, Q_{p+1}, \cdots, Q_{n}
$$

where $Q_{r} \subset \mathscr{X}_{r}=\left\{\chi \in \mathscr{X}^{(n)}(A) \mid \operatorname{dim} \chi=r\right\}$. First define $Q_{p}$ to be a compact neighborhood of $\chi_{T}$ (in $\left.\mathscr{X}^{(n)}(A)\right)$ which is contained in $\mathscr{X}_{p} \cap \mathscr{E}_{\mathscr{E}}$ and such that

$$
\mathscr{Y}\left(Q_{p}\right) \subset W_{0}
$$

this is possible by Corollary 1 of Proposition 12 and Lemma 13.

Now assume that $Q_{p}, \cdots, Q_{r}$ have already been defined (where $r<n$ ) in such a way that $Q_{j} \subset \mathscr{X}_{j}$ and $Q_{j}$ is a compact neighborhood of $\mathscr{Y}_{T} \cap \mathscr{X}_{j}$ $(j=p, \cdots, r)$. Let $D_{r+1}$ be the family of all $\chi$ in $\mathscr{X}_{r+1}$ which (a) are not irreducible, and (b) have no irreducible components lying in $\boldsymbol{Q}_{p} \cup Q_{p+1}$ $\cup \cdots \cup Q_{r}$.

I claim that the closure of $D_{r+1}$ does not intersect $\mathscr{Y}_{T}$.

Indeed, suppose $\chi_{i} \rightarrow \chi \in \mathscr{Y}_{T}$ weakly*, where each $\chi_{i} \in D_{r+1}$. By Lemma 14 we can pass to a subnet and assume that each $\chi_{i}$ has an irreducible component $\phi_{i}$ such that $\phi_{i} \rightarrow \phi \in \mathcal{Y}_{T}$ weakly*. Since $\chi_{i}$ is not irreducible, $\phi_{i}(1)$ $\leqq r$ and so $p \leqq \operatorname{dim} \phi \leqq r$. Since $\phi_{i}$ lies $i$-eventually in $Q_{(\operatorname{dim} \phi)}$, we have contradicted condition (b) of the definition of $D_{r+1}$. So the claim is proved.

We now define $Q_{r+1}$ to be a compact neighborhood of $\mathscr{Y}_{T} \cap \mathscr{X}_{r+1}$ contained in $\mathscr{X}_{r+1}$, such that

$$
\begin{aligned}
& Q_{r+1} \cap D_{r+1}=\Lambda, \\
& \mathcal{Y}\left(Q_{r+1}\right) \subset W_{0} .
\end{aligned}
$$

The first of these conditions can be satisfied by virtue of the above claim, the second by using the same argument as in (I) of the proof of Lemma 13.

The definition of $Q_{p}, \cdots, Q_{n}$ is now complete. Set

$$
M=\left\{S \in \hat{A}^{(n)} \mid \chi_{S} \in Q_{p} \cup Q_{p+1} \cup \ldots \cup Q_{n}\right\} .
$$


We shall show that $M$ is a compact neighborhood of $T$ contained in $U(W)$. By an earlier comment this will complete the proof.

That $M \subset U(W)$ follows from the fact that each $Q_{j} \subset W$ (see (19) and (21)).

To show that $M$ is a neighborhood of $T$, suppose that $\chi_{T_{i}} \rightarrow \phi$, where $\chi_{T} \prec \phi \in \mathscr{X}_{r}(r=p, \cdots, n)$. Since $Q_{r}$ is a neighborhood of $\mathscr{Y}_{T} \cap \mathscr{X}_{r}$, we have $\chi_{T_{i}} \in Q_{r}$, and hence $T_{i} \in M$, for all large $i$. It follows from Theorem 3 that $M$ is a neighborhood of $T$.

Finally we shall show that $M$ is compact. Let $\left\{T_{i}\right\}$ be a net of elements of $M$. We may pass to a subnet and assume that $\chi_{T_{i}} \in Q_{r}$ for all $i$ $(r=p, \cdots, n)$. Since $Q_{r}$ is compact, we can again pass to a subnet and assume that $\chi_{T_{i}} \rightarrow \phi \in Q_{r}$. Now, if $\phi$ is irreducible, then $\phi=\chi_{S}$ where $S \in M$; and $T_{i} \rightarrow S$. Assume that $\phi$ is not irreducible. Since $Q_{p} \subset \mathscr{E}$, we have $r>p$. Since $Q_{r}$ is disjoint from $D_{r}$ (by (20)), one of the irreducible components of $\phi$, say $\chi_{s}$, lies in $Q_{p} \cup \cdots \cup Q_{r-1}$. But this implies that $T_{i} \rightarrow S \in M$. Thus, whether $\phi$ is irreducible or not, there is a subnet of $\left\{T_{i}\right\}$ converging to an element of $M$. Hence $M$ is compact.

10. *-representations of a Banach *-algebra. Suppose that $A$ is a Banach *-algebra (satisfying $\left\|x^{*}\right\|=\|x\|$ ). By a ${ }^{*}$-representation of $A$ we mean of course a representation $T$ of $A$ in a Hilbert space, such that, for each $a$, $T(a)$ is a bounded operator and $T\left(a^{*}\right)=(T(a))^{*}$. Let $A_{u n}^{f}$ be the family of all unitary equivalence classes of finite-dimensional irreducible ${ }^{*}$-representations of $A$. Schur's Lemma tells us that two elements of $\hat{A}_{u n}^{f}$ which are equivalent are also unitarily equivalent. Thus $\hat{A}_{u n}^{f}$ may be regarded as a subset of $\hat{A}^{f}$. Let $\hat{A}_{u n}^{(n)}=\hat{A}^{(n)} \cap \hat{A}_{u n}^{f}$.

For each $a$ in $A$ let $\|a\|_{c}=\sup _{T}\|T(a)\|$, where $T$ runs over all *-representations of $A$. Forming the closed two-sided ${ }^{*}$-ideal $N=\left\{a \mid\|a\|_{c}=0\right\}$, and completing the ${ }^{*}$-algebra $A / N$ with respect to the norm \|\|$_{c}$ lifted to $A / N$, we obtain a $C^{*}$-algebra $B$ called the $C^{*}$-completion of $A$. The ${ }^{*}$-representations of $A$ and of $B$ are in perfect one-to-one correspondence in a natural and well-known manner. Restricted to $\hat{A}_{u n}^{f}$, this correspondence will be called $F$. Since by Theorem $1 \hat{B}_{u n}^{f}=\hat{B}^{f}, F$ is a one-to-one mapping of $\hat{A}_{\text {un }}^{f}$ onto $\hat{B}^{f}$.

Proposition 15. For each positive integer $n, \hat{A}_{u n}^{(n)}$ is a closed subset of $\hat{A}$, and $F \mid \hat{A}_{u n}^{(n)}$ is a homeomorphism of $\hat{A}_{u n}^{(n)}$ onto $\hat{B}^{(n)}$.

Proof. Let us identify $(A / N)^{-}$with a closed subset of $\hat{A}$ (Remark 2 following Proposition 3). Then $\hat{A}_{u n}^{(n)} \subset(A / N)^{-}$. In fact, by Theorem $1, \hat{A}_{u n}^{(n)}$ is just the set of all restrictions to $A / N$ of elements of $\hat{B}^{(n)}$. So our result follows from Proposition 14 and the remark immediately preceding it.

Questions. Is $\hat{A}_{u n}^{f}$ closed in $\hat{A}^{f}$ ? Is $F$ a homeomorphism of $\hat{A}_{u n}^{f}$ onto $\hat{B}^{f}$ ? 


\section{Bibliography}

1. C. W. Curtis and I. Reiner, Representation theory of finite groups and associative algebras, Interscience, New York, 1962.

2. J. Dixmier, Sur les $C^{*}$-algebres, Bull. Soc. Math. France 88 (1960), 95-112.

3. __ Points sépares dans le spectre $d^{\prime}$ une $C^{*}$-algébre, Acta Sci. Math. (Szeged) 22 (1961), 115-128.

4. J. M. G. Fell, The dual spaces of $C^{*}$-algebras, Trans. Amer. Math. Soc. 94 (1960), 365-403.

5.,$C^{*}$-algebras with smooth dual, Illinois J. Math. 4 (1960), 221-230.

6. - The structure of algebras of operator fields, Acta Math. 106 (1961), 233-280.

7. J. Glimm and R. V. Kadison, Unitary operators in $C^{*}$-algebras, Pacific J. Math. 10 (1960), 547-556.

8. R. Godement, A theory of spherical functions. I, Trans. Amer. Math. Soc. 73 (1952), 496-556.

9. N. Jacobson, $A$ topology for the set of primitive ideals in an arbitrary ring, Proc. Nat. Acad. Sci. U. S. A. 31 (1945), 333-338.

10. , Structure of rings, Amer. Math. Soc. Colloq. Publ. Vol. 37, Amer. Math. Soc., Providence, R. I., 1956.

11. R. V. Kadison, Irreducible operator algebras, Proc. Nat. Acad. Sci. U. S. A. 43 (1957), 273-276.

12. G. W. Mackey, Infinite-dimensional group representations, Bull. Amer. Math. Soc. 69 (1963), 628-686.

13. A. E. Taylor, A geometric theorem and its application to biorthogonal systems, Bull. Amer. Math. Soc. 53 (1947), 614-616.

14. J. Wermer, Banach algebras and analytic functions, Advances in Math. 1 (1961), fasc. 1, 51-102.

\section{UNIVERSITY OF WASHINGTON, Seattle, Washington}

